\title{
Effect of sucrose and propylene glycol on the vitrification of sheep oocytes
}

\author{
Dike I. P.* and Obembe 0. 0 . \\ Department of Biological Sciences, Covenant University, Ota, Ogun State, Nigeria. \\ Accepted 11 January, 2013
}

\begin{abstract}
Vitrification solutions containing both permeating and non-permeating cryoprotectants provide better productions of oocytes during freezing and thus contribute to the survival of oocytes. We have investigated the efficency of sucrose in combination with propylene glycol (PROH) on the cryopreservation of the oocytes at the meoisis II (MII) stage. The vitrification of sheep oocytes was performed using $5 \mathrm{M}$ PROH, PROH in combination with sucrose at varying concentrations (5 M PROH + $0.5 \mathrm{M}$ sucrose/1 $\mathrm{M}$ sucrose/1.5 M sucrose/2 M sucrose) and $1.0 \mathrm{M}$ sucrose. It was concluded that sucrose in combination with propylene gylcol is most efficient at concentration between 1.0 to $1.5 \mathrm{M}$. Sucrose was found to aid in the maintainance of the morphological structure of the oocytes vitrified but at high concentrations sucrose has adverse effect on cleavage. Hence sucrose is best used as an additive cryoprotectant when supporting a permeating cryoprotectant, which maintains the integirty of the internal structures through its permeability.
\end{abstract}

Key words: Cryoprotection, PROH, sucrose, sheep oocytes.

\section{INTRODUCTION}

Vitrification, a recent approach to achieve rapid freezing without the use of a freezing machine is a physical process by which a highly concentrated solution of both permeating and non-permeating cryoprotectants solidified during cooling without ice crystal formation. Cryoprotective additives (CPAs) or cryoprotectants are chemicals that reduce the injury to cells during freezing and thawing. These cryoprotectants are needed during the freezing of tissues and cells in order to avoid damage during freezing and thawing. Though useful, these cryoprotectants at inappropriate molar concentration can cause damage to the cells due to osmotic affects and chemical toxicity (Gordon, 1994). During vitrification, cells are osmotically dehydrated by brief exposure to concen-

*Corresponding author. E-mail: ejpdike@gmail.com. Tel: 02348083271422.

Abbreviations: CPA, cryo-protective agents; PG/PROH, propylene glycol; EG, ethylene glycol; DMSO, dimethyl sulfoxide; TL HEPES, HEPES buffered Tyrode's Lactate; TALP, Tyrode's lactate solution; PBS, phosphate buffered saline (containing $\mathrm{Ca}^{2+}$ and $\mathrm{Mg}^{2+}$ ); FCS, fetal calf serum. trated solution of cryoprotectants (less than $6 \mathrm{M}$ ) prior to cooling to $-196^{\circ} \mathrm{C}$. The solid cryoprotectant retains the normal molecular and ionic distribution of the liquid state and is considered to be an extremely viscous super cooled liquid, whose property can be enhanced in the presence of macromolecules (Niemann, 1991),

Conventional cryoprotectants are glycols (alcohols containing at least two hydroxyl groups), such as ethylene glycol, propylene glycol (PROH) and glycerol. Other cryoprotectants like dimethyl sulfoxide (DMSO), sucrose, trehalose and ficoll have also been used. DMSO is one of the most widely used conventional cryoprotectant. glycerol and DMSO have been used for decades by cryobiologists to reduce ice formation in cattle sperm and embryos that are cold-preserved in liquid nitrogen. However in the late 1990s, PROH has also been used (Gordon, 1994, Dike, 2009).

Dike (2009) vitrified sheep oocytes using ethylene glycol, PROH and DMSO at concentrations $5.5+1 \mathrm{M}$ sucrose, $5.0+1.5 \mathrm{M}$ sucrose and $4.5+0.5 \mathrm{M}$ sucrose, respectively and found $\mathrm{PROH}$ to be the more effective cryoprotectant when combined with the vitrification technique thereby yielding a higher rate of cleavage of $28 \%$ in vitro. 
Table 1. Solutions employed for the vitrification of oocytes.

\begin{tabular}{lll}
\hline S.N & Solution & Concentration \\
\hline 1 & Vitrification I & $5.0 \mathrm{M}$ Propylene glycol \\
2 & Vitrification II & $5.0 \mathrm{M}$ Propylene glycol $+0.5 \mathrm{M}$ sucrose \\
3 & Vitrification III & $5.0 \mathrm{M}$ Propylene glycol $+1.0 \mathrm{M}$ sucrose \\
4 & Vitrification IV & $5.0 \mathrm{M}$ Propylene glycol $+1.5 \mathrm{M}$ sucrose \\
5 & Vitrification V & $5.0 \mathrm{M}$ Propylene glycol $+2.0 \mathrm{M}$ sucrose \\
6 & Vitrification VI & $1.0 \mathrm{M}$ Sucrose \\
\hline
\end{tabular}

From the previous studies on vitrification, it was evident that there seems to be a correlation between cleavage rate and sucrose in the vitrification solution. Hence, we have investigated further, in this study, the effect of sucrose on vitrification in the presence of $\mathrm{PROH}$.

\section{MATERIALS AND METHODS}

\section{Sources, in vitro culture and maturation of oocytes}

Ovaries of mature ewes from India were collected immediately after slaughter from the regional (India) abattoir in normal saline supplemented with $10 \mu \mathrm{L}$ gentamycin per $1 \mathrm{ml}$ and transported at $37^{\circ} \mathrm{C}$ to the laboratory in vacuum flask within one hour. Upon arrival at the laboratory, the ovaries were removed of extra ovarian tissues and washed two to three times with tap water and seven to eight times with $0.9 \%$ normal saline at 37 to $38^{\circ} \mathrm{C}$. The ovaries were then kept in a sterile beaker containing normal saline $\left(37\right.$ to $38^{\circ} \mathrm{C}$ ) supplemented with $10 \mu \mathrm{L}$ gentamycin per $1 \mathrm{ml}$.

The ovaries were sliced as per the standard slicing technique described by Dutta et al. (1993) and the follicular fluid collected was then transferred into $50 \mathrm{ml}$ sterile conical tube and kept at $37^{\circ} \mathrm{C}$ in water bath $(10 \mathrm{~min})$ for the oocytes to settle at the bottom due to gravity. The supernatant was then discarded and the pellet was diluted with HEPES buffered Tyrode's Lactate (TL HEPES) and transferred to two to three, $90 \mathrm{~mm}$ Petri dishes for oocyte screening and graded.

The oocytes were then graded according to their morphology (Gordon and Lu, 1995): Grade A: Good+: Compact multi-layered cumulus with greater than three layers and a homogeneous ooplasm. Grade B: Good: Compact cumulus of one to three layers with homogeneous ooplasm having a coarse appearance and a darker zona pellucida.

Grades A and B oocytes (18 to 20 oocytes/droplets) were then transferred to pre-incubated maturation droplets. The oocytes were allowed to mature in vitro for 24 to $26 \mathrm{~h}$ in $\mathrm{CO}_{2}$ incubator under $5 \%$ $\mathrm{CO}_{2}, 95 \%$ relative humidity and $38.5^{\circ} \mathrm{C}$ temperature. After $24 \mathrm{~h}$ of in vitro culture of oocytes, the oocytes were examined under the zoom stereomicroscope and assessed for cumulus layer expansion.

\section{Cryopreservation of MII stage oocytes}

Cryopreservation of oocytes at the meoisis II (MII) stage was performed using $5 \mathrm{M} \mathrm{PROH}, \mathrm{PROH}$ in combination with sucrose at varying concentrations (5 M PROH+0.5 M sucrose/1 M sucrose/1.5 $\mathrm{M}$ sucrose/2 $\mathrm{M}$ Sucrose) and $1.0 \mathrm{M}$ sucrose by the vitrification method (Table 1). The oocytes were vitrified and stored for three weeks. The oocytes were then placed in drops of freezing media (phosphate buffered saline with $\mathrm{Ca}^{2+}$ and $\mathrm{Mg}^{2+}$ (phosphate buffered saline, $\mathrm{PBS})+5 \%$ fetal calf serum (FCS) + vitrification solution) containing the cryoprotectant and left $45 \mathrm{~s}$ for equilibration.

\section{Loading of the straws}

After equilibration, the oocytes were immediately loaded into the middle of a $0.25 \mu$ l French mini straw. First $60 \mu \mathrm{l}$ of the $1.5 \mathrm{M}$ of sucrose was aspirated into the straw followed by $5 \mathrm{~mm}$ air space, then $40 \mu \mathrm{l}$ of freezing media (PBS $+5 \%$ FCS + vitrification solution) containing cryoprotectant and 20 to 25 oocytes, followed by $5 \mathrm{~mm}$ air space and finally $60 \mu \mathrm{l}$ of $0.5 \mathrm{M}$ sucrose. The open end of the straw was sealed. The entire operations were carried out at ambient temperature. A total of 350 oocytes were frozen and studied for each group.

\section{Freezing of straws}

After filling, the sealed straws were slowing introduced to the liquid nitrogen vapours for $5 \mathrm{~min}$ and then dipped slowly into liquid nitrogen $\left(\mathrm{LN}_{2}\right)$ within $45 \mathrm{~s}$ and transferred to $\mathrm{LN}_{2}$ goblets and stored at $-196^{\circ} \mathrm{C}$ in $\mathrm{LN}_{2}$ storage containers for three weeks (Figure 1).

\section{Post warming morphological evaluation of vitrified warmed oocytes}

After three weeks, the oocytes were thawed after removal of cryoprotectants, the cumulus oocytes complexes were washed four times in Fertilization Tyrode's lactate solution (TALP) medium and freed from cumulus cells by repeated pipetting. Morphological evaluation was done under zoom stereomicroscope.

Morphologically damaged oocytes which were identified as oocytes with dark granulated or fragmented cytoplasm, indistinct vitelline membrane, widened perivitelline space or fragmented zona pellucida were removed and 10 to 12 morphologically normal oocytes were selected to be placed in the fertilization droplets.

\section{Evaluation of in vitro fertilization of vitrified warmed oocytes}

Testes from adult rams collected from the slaughter house were washed thoroughly, after removing tunica albugenia, with tap water and $0.9 \%$ saline supplemented with gentamycin. The cauda epididymis was sterilized with $70 \%$ alcohol and incised deeply using a surgical blade. Then using a syringe the sperm TALP medium was used to collect the seminal fluid. The semen collected were assessed for motility of the sperms and then processed for sperm separation by Percoll method.

The sperms were layered on the top of the percoll gradient. The tube was then centrifuged at $800 \mathrm{rpm}$ for $30 \mathrm{~min}$. The hazy layer was recovered immediately after centrifugation with a sterile pipette. Sperms were again washed with $10 \mathrm{ml}$ of sperm TALP at $400 \mathrm{rpm}$ for $10 \mathrm{~min}$ and the supernatant was removed until $100 \mu$ l was left in the tube. The motility of the sperms was assessed under lower (10x) magnification of the inverted phase contrast microscope. The concentration of sperms was adjusted to $2 \times 10$ sperms $\mathrm{ml}^{-1}$ using haemocytometer.

The oocytes which deemed morphologically normal were then used in the investigation of fertilizability of the oocytes. The pre incubated fertilization droplets with oocytes were inseminated with sperms at a concentration of two to 10 sperms $\mathrm{ml}^{-1}$. Now two sets of oocytes were incubated, that is, freshly harvested oocytes and the thawed oocytes. These oocytes were then incubated with the sperms for 18 to $20 \mathrm{~h}$ at $38.5^{\circ} \mathrm{C}, 5 \% \mathrm{CO}_{2}$ and $95 \%$ relative humidity. After $24 \mathrm{~h}$ of fertilization, the presumptive zygotes were observed 


\section{Wasfing oocytes were washed in PBS +5\% FCS + Antibiotic}

\section{Defydration Dehydrate oocytes at room temperature for 5 minutes}

\section{Equif6ration Transfer to freezing media (PBS+5\% FCS+ Vitrification solution)}

\section{Coading Straw Load 20-25 oocytes per straw}

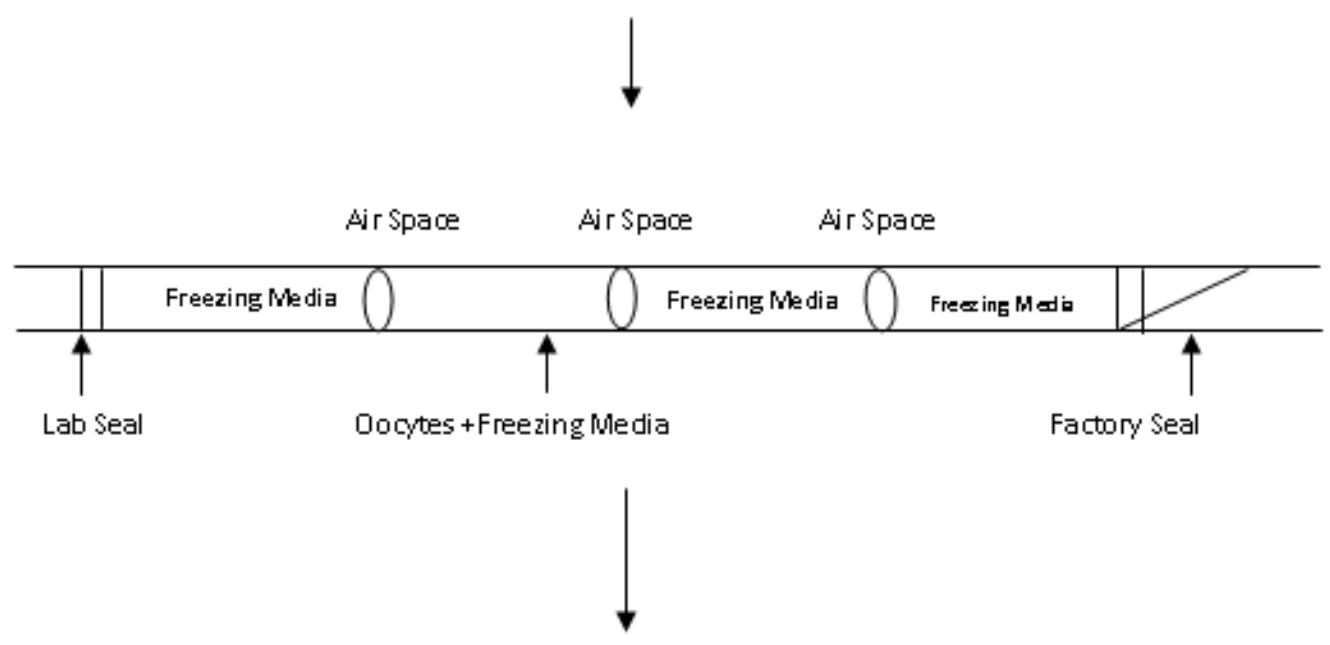

\section{Vitrification Introduce to the LN2 vapours for 5 minutes and then plunge}

Figure 1. Diagrammatic representation of the vitrification protocol. PBS, Phosphate buffered saline (containing $\mathrm{Ca}^{2+}$ and $\mathrm{Mg}^{2+}$ ); FCS, fetal calf serum.

under the zoom stereomicroscope or phase contrast microscope for evidence of cleavage.

\section{Statistical analysis}

Efficiency of the five vitrification solutions were analyzed using the student's $t$ test using the INSTAT software and $\mathrm{P}<0.05$ was said to be significant.

\section{RESULTS}

All the good quality oocytes (A and B) recovered through the slicing technique were utilized for in vitro maturation. The maturation rate obtained was $76 \%$. Assessment of maturation of oocytes was carried out after $24 \mathrm{~h}$ of in vitro culture of oocytes. The oocytes were examined under the microscope and assessed for cumulus cell layer expansion.

Effect of the vitrification solutions (VS) on recovery rate and morphology of oocytes

The post thaw recovery rate obtained for VS I $(5.0 \mathrm{M}$ $\mathrm{PROH}+$ no sucrose), VS II (5.0 M PROH + $0.5 \mathrm{M}$ sucrose), VS III (5.0 M PROH + $1 \mathrm{M}$ sucrose), VS IV (5.0 $\mathrm{M} P R O H+1.5 \mathrm{M}$ sucrose), VS V (5.0 M PROH + $2 \mathrm{M}$ sucrose) and VS VI (1.0 M sucrose) by vitrification method were 81.2, 87.8, 88.14, 92.43, 88.02 and $86.27 \%$, respectively and recovery of morphologically abnormal oocytes of 79.54, 74.05, 69.65, 53.77, 66.25 and $66.05 \%$, respectively (Figure 2 ). The control, noncryopreserved oocytes showed a higher post thaw 


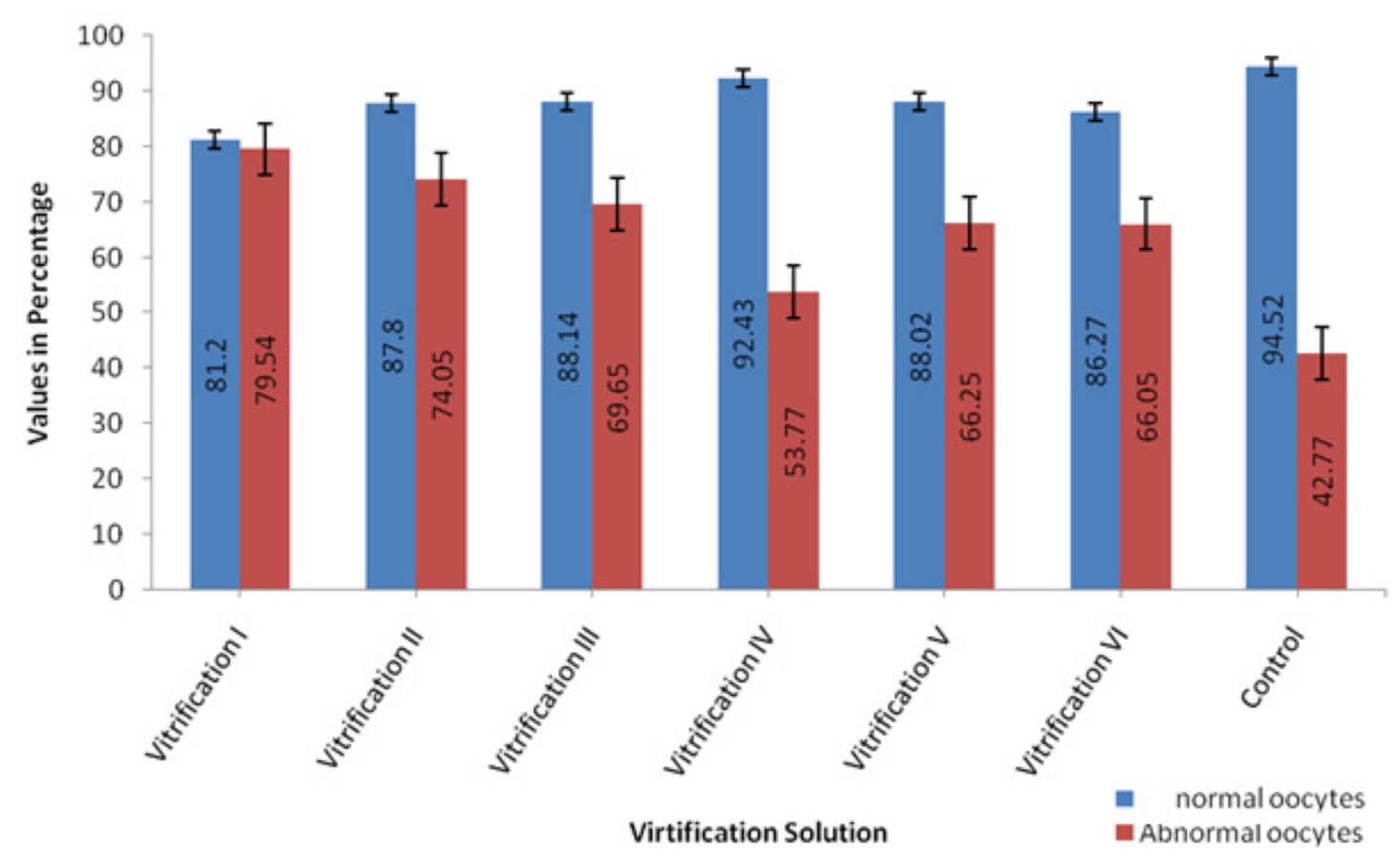

Figure 2. Post thaw recovery rate of morphologically normal and abnormal oocytes for the various vitrification solutions.

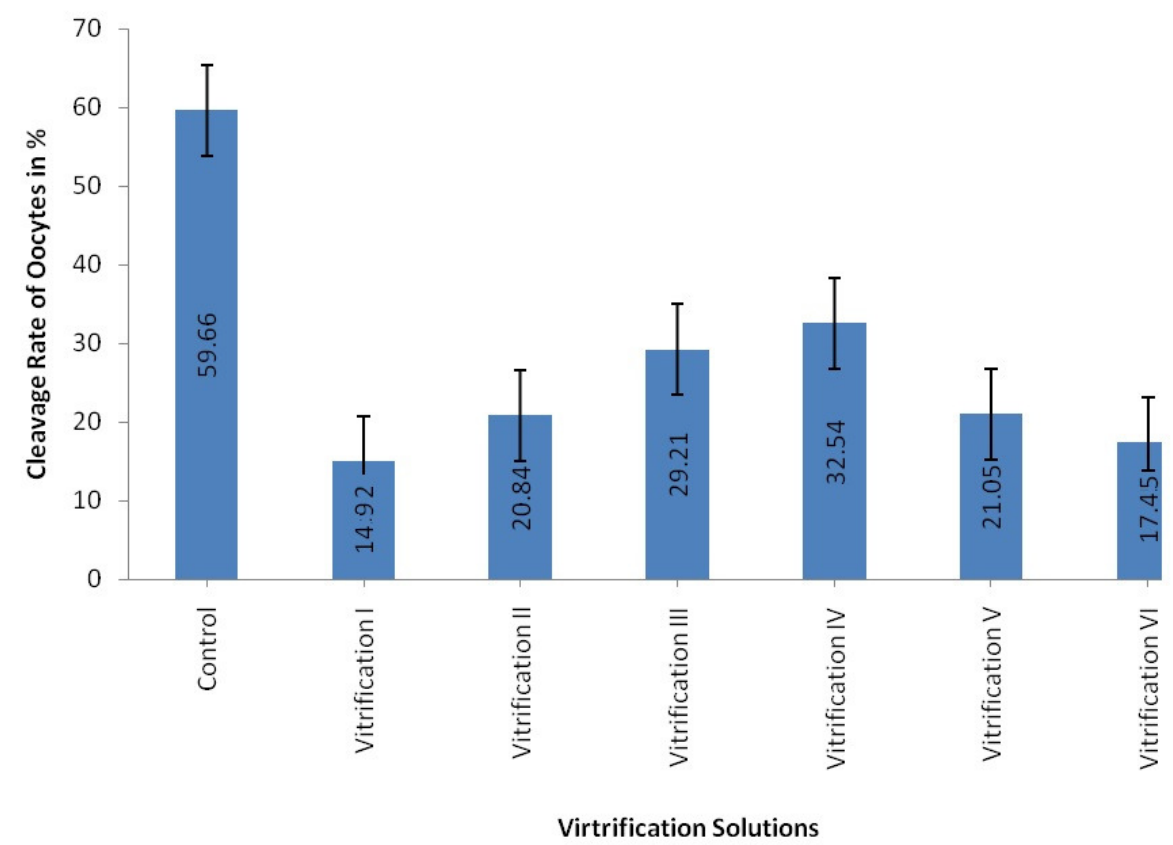

Figure 3. Cleavage rate of oocytes for the various vitrification solutions.

recovery rate of $94.52 \%, 42.77 \%$ of which were morphologically abnormal.

Oocytes were assessed for cleavage and developmental competence for seven to eight days post insemination in vitro. The developmental competence was evaluated by assessing the cleavage rate. On fertilization, the cleavage rates and the percentage oocytes recovery were observed significantly higher in the oocytes frozen in the vitrification solution III and IV (Figure 3). 


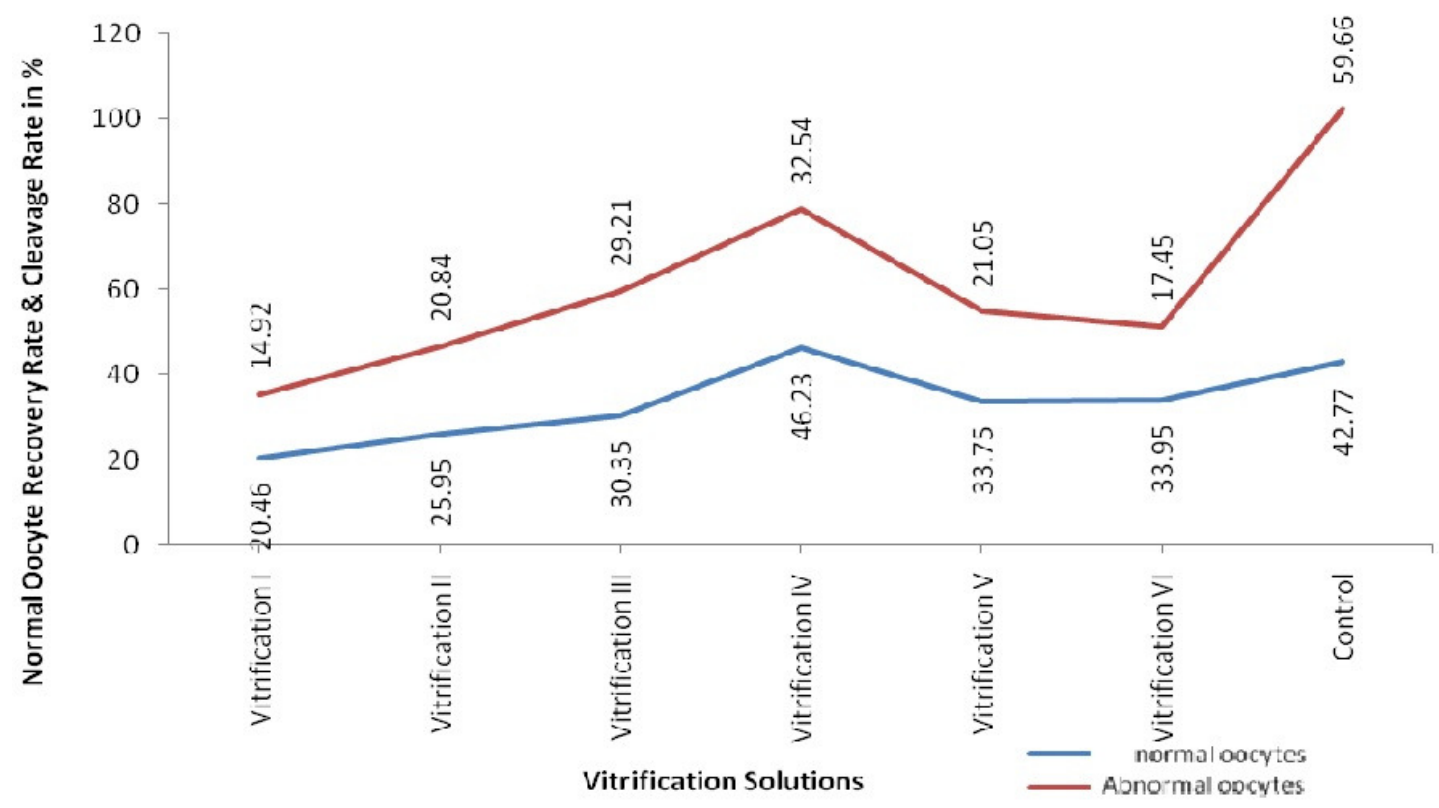

Figure 4. Comparison of efficiency of the various vitrification solutions.

Thus, the percentage of cleavage rate of oocytes frozen by vitrification method using vitrification solutions I, II, III, IV, $\mathrm{V}$ and $\mathrm{VI}$ were 14.92, 20.84, 29.21, 32.54, 21.05 and $17.45 \%$, respectively (Figure 3 ). However, the freshly collected oocytes showed a mean cleavage rate of $59.66 \%$ which was significantly higher than that of the vitrified oocytes.

Figure 4 shows a comparison of the efficiency of the various vitrification solutions. The results indicate that $\mathrm{PROH}$ on its own did not provide good developmental rates $(14.92 \%)$ but when combined with sucrose, the rate of development was increased ranging from 20.84 to $32.54 \%$.

\section{DISCUSSION}

Controlled freezers that freeze biological samples using programmable steps, or controlled rates, have been used all over the world for human, animal and cell biology to better preserve the sample in liquid nitrogen. Although, this process is time consuming and expensive. Vitrification, which is a relatively new technique combines benefits such as ease of use and cost-effectiveness to implement methodology with flexibility in terms of transportability to the field for samples which require immediate cryopreservation. Vitrification usually requires the addition of cryoprotectants prior to cooling. The cryoprotectants act like antifreeze: they lower the freezing temperature. They also increase the viscosity, thus instead of crystallizing, the solution turns into an amorphous ice, that is, it vitrifies (Bhat et al., 2005).

The phenomena, which can cause damage to cells during cryopreservation are solution effects, extracellular ice formation, dehydration and intracellular ice formation. Many of these effects can be reduced by the use of effective cryoprotectants. When tissues are cooled slowly, water migrates out of cells and ice forms in the extracellular space. Too much extracellular ice can cause mechanical damage to the cell membrane due to crushing. The migration of water causing extracellular ice formation can also cause cellular dehydration. Thus the vitrification solution used should ensure that extracellular ice formationis reduced (Bhat et al., 2005).

Thus, two conditions are required to allow vitrification, these include an increase in the viscosity and a depression of the freezing temperature. Many solutes do both, but larger molecules generally have larger effect, particularly on viscosity. Those solutes that do, such as dimethyl sulfoxide, are often toxic in high concentration. Thus, mixtures of cryoprotectants have less toxicity and are more effective than single-agent cryoprotectants. A mixture of formamide with DMSO, $\mathrm{PROH}$ and a colloid was for many years the most effective of all artificially created cryoprotectants (Bhat et al., 2005), thus cryoprotectant mixtures have been used for vitrification.

From the study, it is evident that sucrose on its own is not an efficient cryoprotectant but when it is combined with an efficient cryoprotectant such as $\mathrm{PROH}$, it provides a good medium for vitrification. Thus, it may be due to the fact that sucrose on its own is non-permeating and solute must penetrate the cell membrane in order to achieve increased viscosity and depressed freezing temperature inside the cell It should be noted also that PROH on its own did not provide good developmental rates (14.92\%) but when combined with sucrose, the rate of develop- 
ment was increased ranging from 20.84 to $32.54 \%$ (Figure 4). This can be attributed to the fact that extracellular ice formation would be higher if $\mathrm{PROH}$ is used and thus causing cell damage and reduce rate of development.

Previous experiments conducted on the vitrification of in vitro matured bovine oocytes, showed that the concentration of sucrose $(1$ and $0.67 \mathrm{M})$ in vitrification solutions consisting of a cryoprotectant (ethylene glycol and sucrose) gave rise to the cleavage rates of 27.7 and $38.0 \%$, respectively. This attested to the fact that $0.67 \mathrm{M}$ in vitro fertilization was significantly higher than $1 \mathrm{M}$.

Similarily from the present study it was found that vitification of sheep oocytes using cryoprotectant (propylene glycol and sucrose) showed a high post thaw recovery rate when 1.5 and $2.0 \mathrm{M}$ of sucrose was employed but upon analysing the cleavage rate it was found that cleavage rate was higher for 1.5 and $1.0 \mathrm{M}$ sucrose. These findings are in line with that of Men et al. (1997) who reported that oocytes exposed to vitrification medium which contained only one type of cryoprotectant resulted in very poor post thaw oocytes survival because at higher concentrations it caused membrane damage due to high oocytes swelling. They suggested that a vitrification solution containing both permeating and nonpermeating cryoprotectants provided better productions of oocytes during freezing and thus contributing to the survival of oocytes. This indicated that sucrose helps retain the morphological structure of the oocytes during cryopreservation but there seems to be internal cellular damage caused by the high concentration of sucrose. This is evident from the fact that though the post thaw recovery rate of morphologically normal oocytes was higher when $2.0 \mathrm{M}$ of sucrose was employed, there was a drop in developmental capacity or cleavage rate at that concentration. This is further confirmed by the post thaw recovery rate of normal oocytes when sucrose was used as the vitrification solution, which was $66.05 \%$ but there was a drop in the cleavage rate at the same concentration (17.45\%)
In conclusion, sucrose in combination with $\mathrm{PROH}$ is most efficient at concentrations between 1.5 to $1.0 \mathrm{M}$. Thus further equilibration can be carried out while employing this vitrification solution based on the sample type. It can also be concluded that sucrose aids in maintaining the morphological structure of the oocytes vitrified but at high concentrations has adverse effect on cleavage. Hence, sucrose is suitable for use as an additive cryoprotectant when supporting a permeable cryoprotectant, which maintains the integrity of the internal structures through its permeability.

\section{REFERENCES}

Bhat SN, Sharma A, Bhat SV (2005). Vitrification and glass transition of water: insights from spin probe ESR. Phys Rev Lett 95 (23): 235702. doi:10.1103/PhysRevLett.95.235702. PMID 16384318.

Dike IP (2009). Efficiency of intracellular cryoprotectants on the cryopreservation of sheep oocytes by controlled slow freezing and vitrification techniques. J. Cell Ani. Biol. 3(3):45-49.

Dutta TK, Goswami SL, Das SK (1993).Comparative efficiency of three oocyte recovery methods from sheep ovaries. Indian J. Ani. Sci. 63:1178-1179.

Gordon I (1994). Laboratory Production of Cattle Embryos. Wallingford, UK: CAB international.

Gordon I, Lu KH (1995). Production of embryos in vitro and its impact on livestock production. Theriogenology 33:77-87.

Men HS, Chen JC, Ji WZ, Shang EY, Yang SC, Zou RJ (1997). Cryopreservation of Kunming mouse oocytes using slow cooling, ultrarapid cooling and vitrification protocols. Theriogenology 47:14231431.

Niemann H (1991). Cryopreservation from live stock: current status and research needs. Theriogenology 35:109-124 